\title{
FATORES DE RISCO PARA PNEUMONIA ASPIRATIVA EM CRIANÇAS COM PARALISIA CEREBRAL: REVISÃO INTEGRATIVA
}

FACTORES DE RIESGO PARA NEUMONÍA ASPIRATIVA EN LOS NIÑOS CON PARÁLISIS CEREBRAL: REVISIÓN INTEGRATIVA

RISK FACTORS FOR ASPIRATION PNEUMONIA IN CHILDREN WITH CEREBRAL PALSY: AN INTEGRATIVE REVIEW

Ligia Rodrigues Carneiro Vasconcelos ${ }^{1}$

Roberto Wagner Júnior Freire de Freitas 2

Como Citar:

Vasconcelos LRC, Freitas RWJF.

Fatores de risco para pneumonia aspirativa em crianças com paralisia cerebral: revisão integrativa. Sanare (Sobral, Online). 2021;20(1):91-101.

\section{Palavras-chave:}

Crianças; Paralisia cerebral; Fatores de risco; Pneumonia aspirativa; Assistência domiciliar.

Keywords:

Child; Cerebral Palsy; Risk Factors; Aspiration Pneumonia; Home Nursing.

Palabras clave:

Niños; Parálisis Cerebral; Factores de Riesgo; Neumonía por aspiración; Atención Domiciliaria de Salud.

Submetido: $14 / 04 / 2021$

Aprovado: $10 / 06 / 2021$

Autor(a) para Correspondência: Ligia Rodrigues Carneiro Vasconcelos E-mail: ligiarcarneiro@gmail.com

\section{RESUMO}

Objetivou-se identificar, na literatura científica, os principais fatores de risco para o desenvolvimento de pneumonia aspirativa em crianças portadoras de paralisia cerebral. Trata-se de uma revisão integrativa que incluiu artigos originais indexados às bases de dados Literatura Latino-Americana e do Caribe em Ciências da Saúde (LILACS), Cumulative Index to Nursing and Allied Health Literature (CINAHL), Scopus, Web of Science, MEDLINE/PubMed, Embase e Cochrane, sem restrição de tempo e idioma. Os estudos foram selecionados por meio de diagramas de fluxo Preferred Reporting Items for Systematic Reviews and Meta-Analyses (PRISMA) e apresentados através de um quadro. Foram selecionados 19 artigos publicados em revistas nacionais e internacionais, com predomínio de estudos de coorte prospectivos. Os fatores de risco encontrados foram a aspiração, sialorreia, disfagia, refluxo gastroesofágico, grau $V$ do Sistema de Classificação da Função Motora Grossa, penetração, sintomas respiratórios durante as refeições, sintomas respiratórios frequentes, ronco noturno e convulsão. Os fatores de risco encontrados para pneumonia aspirativa em crianças com paralisia cerebral foram diversos, predominando a aspiração e disfagia. Aponta-se a necessidade de estudos que abordem metodologias preventivas para a pneumonia aspirativa em crianças com paralisia cerebral e abordagens de orientação para cuidadores dessa população em âmbito domiciliar.

1. Fisioterapeuta. Mestranda do Curso de Mestrado Profissional em Saúde da Criança e do Adolescente pela Universidade Estadual do Ceará (UECE). Fisioterapeuta do Hospital Regional Norte de Sobral/CE. E-mail: ligiarcarneiro@gmail.com 0RCID: https://orcid.org/0000-0001-5874-9071

2. Enfermeiro. Doutor em Enfermagem pela Universidade Federal do Ceará (UFC). Pesquisador em Saúde Pública da Fundação Oswaldo Cruz (FIOCRUZ-Ceará) na área de Saúde da Família. E-mail: roberto.wagner@fiocruz.br 0RCID: https://orcid.org/0000-0001-9295-1177

Cert. de Redação Científica: Central das Revisões. Edição de texto: Karina Maria M. Machado. Revisão de provas: Texto definitivo validado pelos(as) autores(as). 


\section{ABSTRACT}

The object of this study is to identify in the scientific literature the main risk factors for the development of aspiration pneumonia in children with cerebral palsy. This is an integrative review that included original articles indexed to the databases Literatura Latino-Americana e do Caribe em Ciências da Saúde (LILACS), Cumulative Index to Nursing and Allied Health Literature (CINAHL), Scopus, Web of Science, MEDLINE/PubMed, Embase and Cochrane, without time and language restrictions. The studies were selected using flow diagrams Preferred Reporting Items for Systematic Reviews and Meta-Analyses (PRISMA) and presented using a table. Nineteen articles published in national and international journals were selected, with a predominance of prospective cohort studies. The risk factors found were aspiration, drooling, dysphagia, gastroesophageal reflux, Gross Motor Function Classification System Level $V$, penetration, respiratory symptoms during meals, frequent respiratory symptoms, night snoring and seizures. The risk factors found for aspiration pneumonia in children with cerebral palsy were diverse, with aspiration and dysphagia being the most prevalent. This points out the need for studies that address preventive methodologies for aspiration pneumonia in children with cerebral palsy and guidance approaches for caregivers for this population at home.

\section{RESUMEN}

El objetivo de este estudio fue identificar, en la literatura cientifica, los principales factores de riesgo para el desarrollo de neumonía aspirativa en los niños portadores de parálisis cerebral. Se trata de una revisión integrativa que incluye artículos originales indexados a las bases de datos Literatura Latinoamericana y del Caribe en Ciencias de la Salud (LILACS), Cumulative Index to Nursing and Allied Health Literature (CINAHL), Scopus, Web of Science, MEDLINE/PubMed, Embase y Cochrane, sin restricción de tiempo e idioma. Los estudios fueron seleccionados por medio de diagramas de flujo Preferred Reporting Items for Systematic Reviews and Meta-Analyses (PRISMA) y presentados a través de un cuadro. Fueron seleccionados 19 artículos publicados en revistas nacionales $e$ internacionales, con supremacía de estudios de cohorte prospectivos. Los factores de riesgo encontrados han sido la aspiración, sialorrea, disfagia, reflujo gastroesofágico, Grado V del GMFCS, penetración, sintomas respiratorios durante las comidas, sintomas respiratorios frecuentes, ronquido nocturno y convulsión. Los factores de riesgo encontrados para neumonía aspirativa en los niños con parálisis cerebral fueron diversos, predominando la aspiración y disfagia como más prevalentes. Se apunta la necesidad de estudios que traten metodologías preventivas para la neumonía aspirativa en los niños con parálisis cerebral y enfoques de orientaciones para cuidadores de esa población en el ámbito domiciliario.

\section{INTRODUÇÃO}

A paralisia cerebral $(P C)$ é um grupo heterogêneo de distúrbios causados por uma lesão não progressiva no cérebro em desenvolvimento, que se caracteriza pela deficiência do controle motor e postural, secundária a um dano ao sistema nervoso central (SNC). É a deficiência motora mais frequente na infância e representa um grupo de desordens permanentes do desenvolvimento, do movimento e postura, que ocorre durante o desenvolvimento do cérebro fetal ou infantil, levando ao comprometimento da funcionalidade da pessoa ${ }^{1}$.

Sua classificação diante das alterações motoras pode ser de acordo com o tipo clínico em espástica, discinética, atáxica ou mista, com a forma de apresentação topográfica e distribuição nos membros, dividindo-se em quadriplégico, diplégico e hemiplégico. Conforme o nível de comprometimento motor, pode ser categorizada pelo Sistema de Classificação da Função Motora Grossa (Gross Motor Function Classification System - GMFCS) um teste específico utilizado para classificar mobilidade em cinco níveis que indicam, em ordem decrescente, a independência e funcionalidade da criança com $P C^{2}$.

Em países desenvolvidos, os dados epidemiológicos mostram uma prevalência variável de 1,5 a 2,5 por 1000 nascidos vivos com PC. No Brasil, estima-se uma prevalência de sete casos para cada 1000 nascidos vivos e surgimento aproximado de 30.000 a 40.000 novos casos por ano ${ }^{3}$. 
A causa mais comum de morbidade e mortalidade em crianças com PC está relacionada ao sistema respiratório, que sofre influência direta e indireta dos distúrbios do tônus, da postura e do movimento, provocando um desalinhamento biomecânico e, com isso, desfavorecendo o bom funcionamento das capacidades pulmonares ${ }^{4}$. A literatura mostra que a doença respiratória na paralisia cerebral é multifatorial e pode ser causada por uma combinação de processos crônicos, deficiência muscular, incoordenação respiratória e distúrbios posturais, juntamente com a incapacidade de eliminar secreções de maneira eficaz, resultando em uma limpeza das vias aéreas ineficiente, o que compromete o sistema pulmonar 5 .

Diante desse contexto, é sabido que muito se fala sobre o alto índice de morbidade e mortalidade em crianças com paralisia cerebral devido às pneumonias, que, em sua maioria, são pneumonias por aspiração de líquidos, alimentos, secreções ou resíduo gástrico devido ao refluxo. No entanto, pouco é discutido e relatado sobre os reais fatores de risco para a ocorrência dessas aspirações, além de medidas preventivas para se evitar esses eventos adversos tão comuns e deletérios para a saúde dessa população.

Contudo, observou-se, depois de ampla busca na literatura nas principais bases de dados da saúde internacional e nacional, muitas publicações que envolvem distúrbios de deglutição, motores e posturais em crianças com paralisia cerebral, porém evidenciou-se uma escassez no que se refere aos distúrbios respiratórios e aos fatores de risco relacionados às pneumonias aspirativas.

Em vista disso, este estudo objetivou identificar na literatura científica os principais fatores de risco para o desenvolvimento de pneumonia aspirativa em crianças portadoras de paralisia cerebral. Acredita-se que estudos dessa natureza sejam relevantes, uma vez que contribuem para a prática baseada em evidência (PBE) e subsidiam intervenções para os profissionais de saúde que prestam assistência a essa população.

\section{METODOLOGIA}

Este artigo trata-se de uma revisão integrativa que foi estruturada em seis etapas distintas: 1) identificação do tema e seleção da hipótese ou questão de pesquisa para a elaboração da revisão integrativa; 2) estabelecimento de critérios para a inclusão e exclusão de estudos/amostragem ou busca na literatura; 3) definição das informações a serem extraídas dos estudos selecionados/ categorização dos estudos; 4) avaliação dos estudos incluídos na revisão integrativa; 5) interpretação dos resultados; 6) apresentação da revisão/síntese do conhecimento ${ }^{6}$.

A questão de pesquisa foi elaborada de acordo com a estratégia População Interesse Contexto $(\mathrm{PIC} 0)^{7}$. Considerou-se, assim, a seguinte estrutura: $P$ - crianças com paralisia cerebral; I - fatores de risco; Co - pneumonia aspirativa. Dessa forma, elaborou-se a seguinte pergunta norteadora: Quais são os principais fatores de risco de pneumonia aspirativa em crianças com paralisia cerebral?

0 levantamento bibliográfico foi realizado em outubro de 2020. 0 acesso inicial às bases de dados foi realizado pelo portal de periódicos da Coordenação de Aperfeiçoamento de Pessoal de Nível Superior (CAPES), em área com Internet Protocol (IP) reconhecida, na Universidade Estadual do Ceará (UECE), com o intuito de garantir a busca ampla, em sua totalidade. Foram selecionadas as seguintes bases de dados para a pesquisa: Literatura Latino-Americana e do Caribe em Ciências da Saúde (LILACS); Cumulative Index to Nursing and Allied Health Literature (CINAHL) via with Full Text (EBSCO); Scopus (Elsevier); Web of Science via Coleção Principal (Clarivate Analytics); MEDLINE/PubMed (via National Library of Medicine); Embase (Elsevier) e Cochrane library.

Os critérios de inclusão consistiram em pesquisas sem delimitação de tempo de publicação, sem restrição de idiomas e de método de estudo. Foram excluídas investigações que não apresentavam 0 resumo ou o artigo completo disponivel; revisões sistemáticas e integrativas; publicações repetidas; pesquisas realizadas com adultos; outros distúrbios neurológicos que não incluíssem a paralisia cerebral; pesquisa que não respondesse à questão norteadora do estudo.

Para a busca nas bases de dados foram usadas as palavras-chave "paralisia cerebral"; "fatores de risco" e "pneumonia aspirativa". A partir delas foram selecionados descritores presentes nos Descritores em Ciências da Saúde (DeCS) e seus equivalentes, no idioma inglês, no Medical Subject Headings (MeSH). Os descritores foram combinados entre si com o conector booleano OR, dentro de cada conjunto de termos da estratégia PICo e, em seguida, cruzados com o conector booleano AND. 
Foram selecionados dois cruzamentos distintos, um elaborado pelos autores e outro cruzamento próprio, criado pela base de dados Embase, com o intuito de ampliar a busca dos artigos. 0s cruzamentos utilizados foram: ("risk factors" OR "risk factor" OR causality OR causation) AND ("pneumonia, aspiration" OR "respiratory aspiration") AND ("cerebral palsy") e ("risk factors"/exp OR "risk factors" OR "causality"/exp OR causality OR causation) AND ("pneumonia, aspiration"/exp OR “pneumonia, aspiration" OR "respiratory aspiration"/exp OR "respiratory aspiration") AND ("cerebral palsy"/exp OR “cerebral palsy").

Para a extração e síntese das informações dos estudos selecionados, utilizaram-se dados que os autores identificaram como relevantes mediante às suas práticas clínicas e de pesquisa. Foram extraídas as seguintes informações: periódico, ano de publicação, país, título, autoria, tipo de estudo, nível de evidência, objetivo, resultado e conclusão.

0 nível de evidência foi determinado da seguinte forma: Nível 1a) Revisão sistemática de ensaio clínico randomizado; Nível 1b) Ensaio clínico randomizado individual; Nível 2a) Revisão sistemática de estudos de coorte; Nível 2b) Estudo de coorte individual; Nível 3a) Revisão sistemática de estudos de caso-controle; Nível 3b) Estudo de caso-controle individual; Nível 4) Série de casos; e Nível 5) 0pinião especializada ou pesquisa de laboratório ${ }^{8}$.

Identificaram-se 157 publicações, das quais, após aplicação dos critérios de inclusão e exclusão, foram selecionados para a amostra desta revisão 19 artigos. Não foram incluídos outros estudos após o processo de busca manual. Para a seleção das publicações, seguiram-se as recomendações do Preferred Reporting Items for Systematic Reviews and Meta-Analyses (PRISMA) ${ }^{9}$, conforme apresentado na Figura 1.

Figura 1. Fluxograma de seleção dos estudos primários, elaborado a partir da recomendação PRISMA.

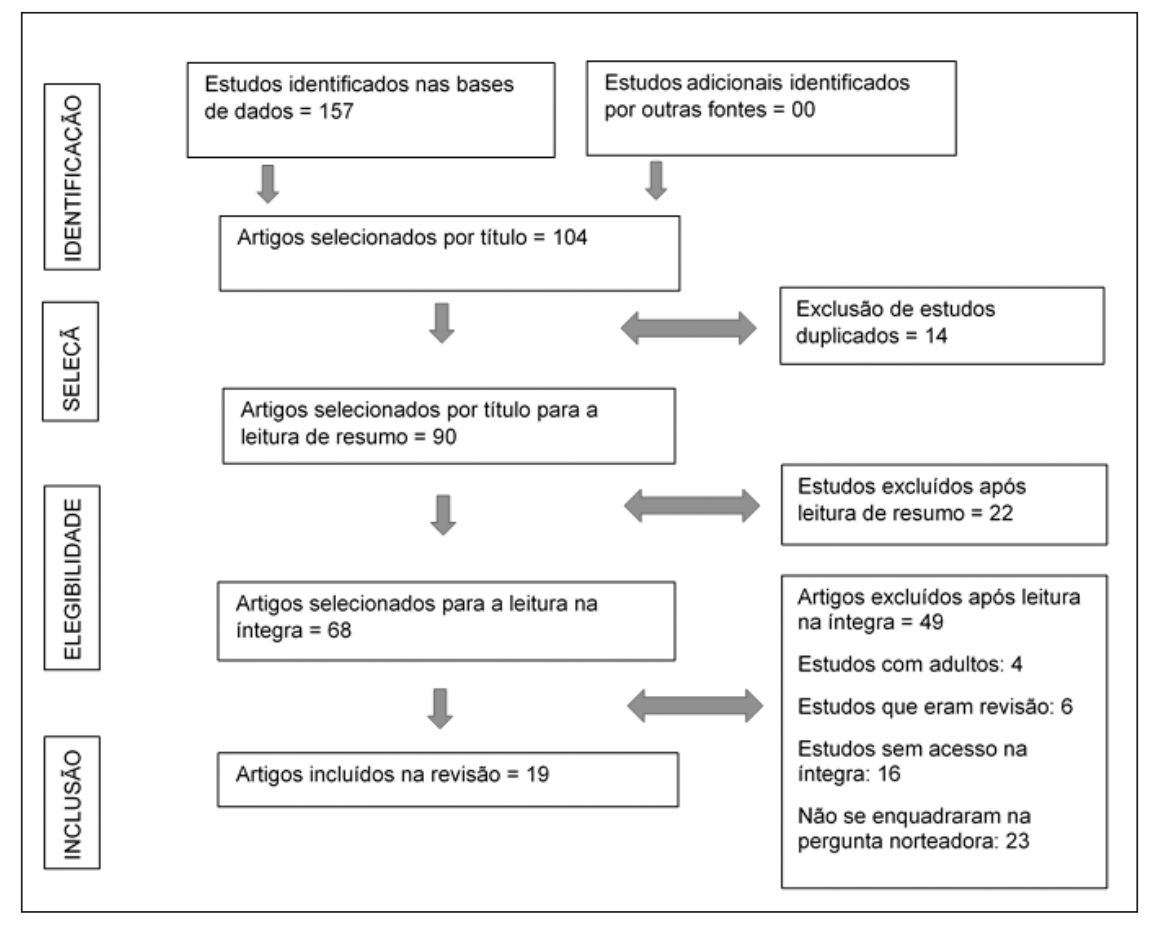

Fonte: Dados da pesquisa.

Por tratar-se de revisão integrativa, a pesquisa não foi submetida ao Comitê de Ética em Pesquisa (CEP), porém foram mantidas as ideias dos autores das publicações utilizadas no desenvolvimento deste estudo.

\section{RESULTADOS}

Nesta revisão foram selecionados 19 artigos, dos quais $14(73,7 \%)$ foram identificados na Pubmed, um $(5,3 \%)$ na LILACS e quatro $(21,1 \%)$ na Web of Science. Desses, $12(63,2 \%)$ tinham sido publicados em periódicos de medicina, cinco $(26,3 \%)$ em revistas interdisciplinares de saúde e dois $(10,5 \%)$ em revistas de outras áreas 
da saúde (fonoaudiologia, medicina e odontologia).

Os artigos sobre a temática começaram a ser publicados no ano de 1994 e tiveram a maior prevalência de tempo de publicação nos anos de 2014 a 2019. Quanto à distribuição territorial, os estudos foram mais frequentes na Austrália $(n=6)$. Os demais estudos foram realizados nos Estados Unidos $(n=2)$, Reino Unido $(n=2)$, Japão $(n=1)$, Turquia $(n=1)$, China $(n=1)$, Israel $(n=1)$, Suécia $(n=1)$, Indonésia $(n=1)$ e Coreia do Sul $(n=1)$. No Brasil, somente dois estudos foram publicados com essa temática, um deles em periódico nacional, em língua portuguesa, e o outro em periódico internacional, em língua inglesa. 0s demais estudos foram publicados em periódicos internacionais, também em língua inglesa.

Em relação ao periódico, dois se destacaram pela quantidade de publicações sobre a temática. 0 International Journal of Pediatric Otorhinolaryngology, considerado Qualis Capes B1 na área interdisciplinar, obteve quatro publicações, e o Archives of Disease in Childhood, considerado Qualis Capes A2 na área medicina I, apresentou duas publicações. Sobre o tipo de estudo dos artigos selecionados, a maioria $(n=12)$ apresentou estudos de coorte, cinco foram estudos retrospectivo e sete foram estudos prospectivos. Um ensaio clínico, um estudo de caso-controle, um estudo transversal, um estudo documental e três estudos de caso. Em relação ao nível de evidência científica, respectivamente, 12 artigos $(63,3 \%)$ receberam nível $2 B$; um $(5,2 \%)$ recebeu nível 1B; um $(5,2 \%)$ nível 3B; um $(5,2 \%)$ obteve nível 4 ; e quatro $(21,1 \%)$, nível 5 .

0 Quadro 1, a seguir, apresenta os principais fatores de risco para pneumonias aspirativas em crianças portadoras de paralisia cerebral, além de algumas informações que foram consideradas importantes, como: ano de publicação, país, tipo de estudo, nível de evidência, resultado e conclusão.

Quadro 1. Caracterização dos estudos selecionados.

\begin{tabular}{|c|c|c|c|c|}
\hline Artigo & Ano/País & Tipo de estudo & $\begin{array}{l}\text { Nivel de } \\
\text { evidência }\end{array}$ & Resultados / Conclusão \\
\hline $\mathrm{A} 1^{10}$ & $\begin{array}{l}2019 \\
\text { Japão }\end{array}$ & Ensaio clínico & Nivel 1B & $\begin{array}{l}\text { A aspiração de saliva está relacionada com } \\
\text { pneumonias aspirativas, de acordo com os achados } \\
\text { nas tomografias de tórax. } 74,1 \% \text { das crianças eram } \\
\text { portadoras de paralisia cerebral. }\end{array}$ \\
\hline $\mathrm{A} 2^{11}$ & $\begin{array}{l}2019 \\
\text { Estados } \\
\text { Unidos }\end{array}$ & Caso-controle & Nivel 3B & $\begin{array}{l}0 \text { fator de risco encontrado foi a aspiração de saliva. } \\
\text { Crianças com paralisia cerebral têm mais chances de } \\
\text { aspiração pulmonar ( } 56 \% \text { positivo no salivograma) } \\
\text { do que jovens adultos saudáveis, e mais risco de } \\
\text { desenvolver pneumonias aspirativas. } \\
0 \text { principal fator de risco associado à pneumonia } \\
\text { aspirativa é a aspiração silenciosa, que afeta } \\
\text { particularmente crianças com paralisia cerebral. }\end{array}$ \\
\hline $\mathrm{A} 4^{13}$ & $\begin{array}{l}2009 \\
\text { Brasil }\end{array}$ & Coorte & Nível 2B & $\begin{array}{l}\text { A disfagia é um fator de risco para a pneumonia } \\
\text { aspirativa em crianças com paralisia cerebral e a } \\
\text { ausculta cervical é uma importante ferramenta para } \\
\text { avaliar a aspiração traqueal. }\end{array}$ \\
\hline$A 5^{14}$ & $\begin{array}{l}2018 \\
\text { Indonésia }\end{array}$ & Coorte & Nivel 2B & $\begin{array}{l}0 \text { estudo considerou o grau de disfunção motora } \\
\text { grossa como um fator de risco para pneumonias } \\
\text { aspirativas em crianças com PC. } 25 \% \text { dos pacientes } \\
\text { do estudo tinham GMFCS grau V, assim como a } \\
\text { disfagia e a aspiração silenciosa. }\end{array}$ \\
\hline$A 6^{15}$ & $\begin{array}{l}2016 \\
\text { Nova York }\end{array}$ & Coorte & Nivel 2B & $\begin{array}{l}0 \text { fator de risco identificado foi a aspiração } \\
\text { crônica. Complementar com a infecção por } \\
\text { Pseudomonas aeruginosa, isolada ou outra bactéria } \\
\text { gram-negativa, ocorreu uma maior frequência de } \\
\text { internações hospitalares, admissões em UTI's e } \\
\text { agravamento da pneumonia. }\end{array}$ \\
\hline
\end{tabular}




\begin{tabular}{|c|c|c|c|c|}
\hline Artigo & Ano/País & Tipo de estudo & $\begin{array}{l}\text { Nivel de } \\
\text { evidência }\end{array}$ & Resultados / Conclusão \\
\hline$A 7^{16}$ & $\begin{array}{l}2009 \\
\text { Brasil }\end{array}$ & Documental & Nivel 5 & $\begin{array}{l}0 \text { fator de risco encontrado foi a aspiração } \\
\text { crônica de saliva. Onde a excisão das glândulas } \\
\text { submandibulares e ligadura dos ductos parótidos } \\
\text { diminuíram a secreção nas vias aéreas superiores } \\
\text { reduzindo a frequência de episódios de pneumonias } \\
\text { aspirativas em crianças com paralisia cerebral. }\end{array}$ \\
\hline$A 8^{17}$ & $\begin{array}{l}2014 \\
\text { Austrália }\end{array}$ & Coorte & Nivel 2B & $\begin{array}{l}\text { o fator de risco encontrado foi a aspiração } \\
\text { salivar crônica devido à sialorreia. } 75 \% \text { das } \\
\text { crianças do estudo tinham paralisia cerebral. } \\
\text { A excisão da glândula sub mandibular com ligadura } \\
\text { da glândula parótida foi um método seguro e } \\
\text { eficaz para controlar o débito salivar em pacientes } \\
\text { com aspiração crônica secundária à sialorreia }\end{array}$ \\
\hline A $9^{18}$ & $\begin{array}{l}2011 \\
\text { Austrália }\end{array}$ & Coorte & Nivel 2B & $\begin{array}{l}\text { A aspiração orofaríngea e aspiração silenciosa foi } \\
\text { encontrada de forma mais prevalente em crianças } \\
\text { com problemas neurológicos como a paralisia } \\
\text { cerebral ( } 21,3 \% \text { das crianças). Fator que predispõe } \\
\text { a pneumonias aspirativas. }\end{array}$ \\
\hline $\mathrm{A} 10^{19}$ & $\begin{array}{l}2006 \\
\text { R e i n o } \\
\text { Unido }\end{array}$ & Coorte & Nivel 2B & $\begin{array}{l}\text { A disfunção oral motora e a aspiração direta são } \\
\text { a principal causa de pneumonia aspirativa em } \\
\text { crianças com neurodeficiência grave ( } 43 \text { das } 57 \\
\text { crianças tinham paralisia cerebral). Pacientes } \\
\text { com gastrostomia tiveram menos ocorrência de } \\
\text { pneumonias. }\end{array}$ \\
\hline $\mathrm{A} 11^{20}$ & $\begin{array}{l}2018 \\
\text { Austrália }\end{array}$ & Coorte & Nivel 2B & $\begin{array}{l}0 \text { grau de disfunção motora grossa nível V, disfagia } \\
\text { orofaríngea, refluxo gastroesofágico, sintomas } \\
\text { respiratórios durante as refeições, sintomas } \\
\text { respiratórios frequentes, ronco noturno e convulsão } \\
\text { são fatores de risco para pneumonias aspirativas } \\
\text { em criança portadora de paralisia cerebral. Sendo } \\
\text { a disfagia orofaríngea o fator de risco mais forte. }\end{array}$ \\
\hline $\mathrm{A} 12^{21}$ & $\begin{array}{l}2016 \\
\text { Turquia }\end{array}$ & Coorte & Nivel 2B & $\begin{array}{l}\text { Tanto os distúrbios faríngeos da deglutição, como } \\
\text { penetração e aspiração, quanto os distúrbios } \\
\text { esofágicos e refluxo, podem resultar em pneumonias } \\
\text { de repetição em pacientes pediátricos. } 83,2 \% \text { das } \\
\text { crianças eram portadoras de paralisia cerebral. }\end{array}$ \\
\hline $\mathrm{A} 13^{22}$ & $\begin{array}{l}2016 \\
\text { Austrália }\end{array}$ & Transversal & Nivel 4 & $\begin{array}{l}0 \text { presente estudo constatou a disfunção oromotora } \\
\text { ou disfagia como principal fator de risco para } \\
\text { pneumonias aspirativas em crianças e jovens com } \\
\text { paralisia cerebral. }\end{array}$ \\
\hline $\mathrm{A} 14^{23}$ & $\begin{array}{l}1997 \\
\text { Israel }\end{array}$ & Estudo de caso & Nível 5 & $\begin{array}{l}0 \text { fator de risco encontrado foi o refluxo } \\
\text { gastroesofágico em bebês e crianças menores de } \\
\text { dois anos, resultando em pneumonias aspirativas } \\
\text { (duas das } 11 \text { crianças do estudo tinham paralisia } \\
\text { cerebral). }\end{array}$ \\
\hline
\end{tabular}




\begin{tabular}{|c|c|c|c|c|}
\hline Artigo & Ano/País & Tipo de estudo & $\begin{array}{l}\text { Nivel de } \\
\text { evidência }\end{array}$ & Resultados / Conclusão \\
\hline $\mathrm{A} 15^{24}$ & $\begin{array}{l}1994 \\
\text { Suécia }\end{array}$ & Coorte & Nivel 2B & $\begin{array}{l}0 \text { refluxo gastroesofágico e a disfagia foram os } \\
\text { fatores de risco encontrados no desencadeamento } \\
\text { de pneumonias aspirativas nos pacientes do estudo. } \\
\text { Observa-se que } 21 \text { dos } 32 \text { participantes tinham } \\
\text { paralisia cerebral. }\end{array}$ \\
\hline$A 16^{25}$ & $\begin{array}{l}2019 \\
\text { Austrália }\end{array}$ & Coorte & Nível 2B & $\begin{array}{l}\text { A hipersalivação é um fator de risco para a } \\
\text { morbidade pulmonar, como a pneumonia aspirativa. } \\
\text { 0nze das quinze crianças do estudo tinham PC com } \\
\text { histórico de internações hospitalares recorrentes } \\
\text { por pneumonia aspirativa. A toxina botulínica } \\
\text { mostrou eficácia no controle de baba dessas } \\
\text { crianças e diminuiu as internações hospitalares por } \\
\text { pneumonias. }\end{array}$ \\
\hline $\mathrm{A} 17^{26}$ & $\begin{array}{l}2013 \\
\text { Reino } \\
\text { Unido }\end{array}$ & Estudo de casos & Nivel 5 & $\begin{array}{l}0 \text { fator de risco encontrado para pneumonia } \\
\text { aspirativa foi a sialorreia ou salivação excessiva, } \\
\text { onde a injeção de toxina botulínica A na glândula } \\
\text { mandibular mostrou uma melhora objetiva e } \\
\text { subjetiva na hipersalivação e, consequentemente, } \\
\text { diminuição de pneumonias aspirativas nos pacientes } \\
\text { com distúrbios neuromusculares, como a paralisia } \\
\text { cerebral. }\end{array}$ \\
\hline $\mathrm{A} 18^{27}$ & $\begin{array}{l}2004 \\
\text { China }\end{array}$ & Estudo de caso & Nivel 5 & $\begin{array}{l}0 \text { fator de risco encontrado para pneumonia } \\
\text { aspirativa no caso da criança com paralisia cerebral } \\
\text { foi a ingestão alimentar por via oral quando o } \\
\text { pescoço estava em posição de extensão. }\end{array}$ \\
\hline$A 19^{28}$ & $\begin{array}{l}2014 \\
\text { Coreia do } \\
\text { Sul }\end{array}$ & Coorte & Nivel 2B & $\begin{array}{l}0 \text { estudo considerou a disfagia como fator de risco } \\
\text { para casos de pneumonia aspirativa. De } 90 \text { crianças, } \\
61 \text { tinham condições neurológicas, das quais } 34 \\
\text { eram portadoras de paralisia cerebral. }\end{array}$ \\
\hline
\end{tabular}

Fonte: Dados da pesquisa.

No que concerne aos resultados dos estudos selecionados, somente um artigo objetivou, especificamente, encontrar os principais fatores de risco para a pneumonia aspirativa em crianças com paralisia cerebral. A maioria relatou esses fatores de risco de maneira complementar a principal assunto da pesquisa ou realizou o estudo em crianças com distúrbios neurológicos variados, de modo que a paralisia cerebral foi incluída. Dos 19 estudos incluídos na revisão, nove $(47,3 \%)$ foram estudos voltados para crianças com paralisia cerebral, três $(15,3 \%)$ foram relacionados a outros distúrbios neurológicos que incluíam crianças com paralisia cerebral e sete $(36,9 \%)$ foram pesquisas que incluíam crianças, objetivando determinar relações de aspiração e distúrbios respiratórios, em que, em sua maioria, tratavam-se de crianças com distúrbios neurológicos, incluindo a paralisia cerebral.

0 fator de risco mais prevalente nesta revisão foi a aspiração, descrita pelos autores como aspiração de saliva ${ }^{10,11}$, aspiração silenciosa ${ }^{12-14}$, aspiração crônica ${ }^{15,16}$, aspiração salivar crônica ${ }^{17}$, aspiração orofaríngea ${ }^{18}$ e aspiração direta ${ }^{19}$. Dez estudos concluíram algum tipo de aspiração nas crianças pesquisadas.

Um dos estudos ${ }^{17}$ relacionou a aspiração salivar crônica com episódios de pneumonia aspirativa devido à sialorreia, relatando que as aspirações foram mais frequentes devido ao aumento salivar na cavidade oral.

0 segundo fator mais predominante relatado foi a disfagia, descrita por alguns autores como disfagia orofaríngea ${ }^{20}$, distúrbios faríngeos da deglutição ${ }^{21}$, distúrbios esofágicos ${ }^{21}$ e disfunção oral motora ${ }^{19,22}$. Nove estudos constataram episódios de pneumonia aspirativa em crianças com PC que tinham disfagia. 
0 refluxo gastroesofágico foi presente em quatro estudos como fator determinante para pneumonias aspirativas $20,21,23,24$.

Três estudos relacionaram a sialorreia como fator de risco $0^{17,25,26}$ e dois identificaram que 0 grau avançado de disfunção motora grossa (Gross Motor Function Classification System - GMFCS) em crianças portadoras de paralisia é um dos fatores de risco para pneumonias aspirativas ${ }^{14,20}$. Nos dois estudos, as crianças foram classificadas com GMFCS nível $V$, considerado o nível mais avançado de comprometimento motor.

Outros fatores de risco foram encontrados, porém com uma menor prevalência, como a penetração ${ }^{21}$, sintomas respiratórios durante as refeições, sintomas respiratórios frequentes, ronco noturno e convulsão ${ }^{20}$.

\section{DISCUSSÃO}

Esta revisão da literatura revelou que os principais fatores de risco para a pneumonia aspirativa em crianças portadoras de paralisia cerebral são: aspiração, sialorreia, disfagia, refluxo gastroesofágico, Grau V do GMFCS, penetração, sintomas respiratórios durante as refeições, sintomas respiratórios frequentes, ronco noturno e convulsão.

A aspiração é um fator conhecido por especialistas clínicos e estudiosos que vivenciam o cotidiano de crianças com PC, sendo um assunto muito discutido na literatura científica e presente nos artigos desta revisão como o causador mais determinante para a pneumonia aspirativa na população investigada. Essas aspirações podem ser de saliva, líquidos, alimento ou resíduo gástrico por consequência de refluxo gastroesofágico. Um ensaio clínico investigou a correlação de pneumonias diagnosticadas em tomografia de tórax com a presença ou ausência de aspiração, em crianças $(74,1 \%$ com PC), e evidenciou que a aspiração de saliva está relacionada às pneumonias aspirativas ${ }^{10}$

Outro estudo constatou aspiração por meio do salivograma, um método cintilográfico para detectar aspiração de saliva, em que foi demonstrada aspiração em $56 \%$ das crianças com paralisia cerebral, em comparação com $0 \%$ dos adultos saudáveis, comprovando que crianças com PC aspiram volumes maiores de saliva durante 0 sono do que adultos sãos ${ }^{11}$.
A história natural da aspiração silenciosa em crianças foi investigada e associada a comorbidades neurológicas, principalmente paralisia cerebral e convulsões ${ }^{12}$. Outro estudo que investigou também a aspiração silenciosa obteve resultados semelhantes, mostrando que crianças com aspiração silenciosa eram mais propensas a ter distúrbios neurológicos como PC18.

$\mathrm{Na}$ paralisia cerebral, a má coordenação é responsável pela ocorrência de disfagia e aspiração direta sequencial. Alguns estudos desta revisão incluíram a disfagia e/ou a aspiração direta como fator de risco para a pneumonia aspirativa ${ }^{19,21,24-28}$.

Em um estudo realizado no ano de 2016, verificou-se que cerca de $43 \%$ das crianças com paralisia cerebral apresentavam disfagia e que, das 24 crianças com disfagia grave, 14 apresentavam pneumonia recorrente ${ }^{29}$. Estes dados reforçam os achados dos artigos desta revisão, que encontraram associação de pneumonias aspirativas em crianças disfágicas com paralisia cerebral.

No que diz respeito à disfunção motora grossa, a literatura relaciona sua diminuição, alterações dos padrões de movimento e limitação da capacidade funcional com maior chance de disfagia em crianças portadoras de PC com graus de III a V, dificuldades alimentares e piora na evolução da disfagia com o passar dos $\operatorname{anos}^{30}$. Nesta revisão, dois estudos relacionaram maior prevalência de pneumonia aspirativa em crianças com GMFCS grau $\mathrm{V}^{18,26}$.

A prevalência da sialorreia em crianças com paralisia cerebral foi revisada em 2016, constatandose a presença de $10 \%$ a $58 \%$ nas crianças com paralisia cerebral, relacionando sua causa com a disfagia e os distúrbios de sensibilidade intraoral ${ }^{31}$. 0 s estudos que compuseram esta revisão mostraram resultados semelhantes, demonstrando alto índice de sialorreia, em uma média de $73 \%$ a $75 \%$, nas crianças com paralisia cerebral ${ }^{17,25-26}$.

A doença do refluxo gastroesofágico (DRGE) é uma condição de alta prevalência na população em geral e bastante presente em pacientes portadores de paralisia cerebral onde vários aspectos contribuem para o seu surgimento, como a redução do esfíncter esofágico, esvaziamento gástrico retardado, motilidade esofágica prejudicada, postura inadequada, convulsões, escoliose, terapia medicamentosa variada, alterações musculares devido a hipotonia muscular ou espasticidade levando a uma deglutição ineficaz podendo causar aspiração traqueal e, consequentemente, 
pneumonia aspirativa ${ }^{32}$. Sua prevalência é citada como $70 \%$ a $90 \%$ em crianças com PC e deficiência de crescimento ${ }^{33}$.

A prevalência encontrada de refluxo gastroesofágico nos estudos incluídos nesta revisão foi de $78,1 \%$, entrando em concordância com os valores achados no estudo de Rogers, realizado no ano de $2004^{33}$.

Verificou-se, em pesquisa realizada em 2017, que pacientes com PC apresentam alterações como bradipneia, expansibilidade torácica diminuída, ausculta atípica e tosse não produtiva, fatores que favorecem 0 aparecimento de patologias como a pneumonia. 0 estudo supramencionado aponta ainda sobre a importância de medidas preventivas e intervenções precoces frente às possiveis complicações respiratórias que crianças portadoras de PC podem apresentar, reforçando que os distúrbios pulmonares são a principal causa de mortalidade nessa população ${ }^{34}$.

É visto, na prática clínica e na literatura, que crianças com distúrbios neurológicos tendem a ter mais internações hospitalares do que crianças saudáveis, sendo essas internações prolongadas e com grande risco de complicação, trazendo custos e falta de rotatividade de leitos de internação ao sistema de saúde. Neste sentido, crianças com condições crônicas necessitam de um tempo de internação maior, quando comparadas às crianças que não possuem tais comorbidades. Demonstrando a importância de medidas preventivas e de políticas públicas para essa população, bem como de apoio aos profissionais e serviços da atenção primária a saúde, incluindo ações que busquem melhorar a qualidade de vida dessa população e medidas preventivas que auxiliem na diminuição de internações hospitalares ${ }^{35}$.

Tendo em vista as diversas comorbidades e distúrbios respiratórios apresentados por crianças com paralisia cerebral relatados nesta revisão, ressalta-se a importância da inserção da família na rede de cuidados e na compreensão desta sobre sua responsabilidade na prevenção de doenças, desempenhando um papel de continuidade nos cuidados com o paciente.

Ressalta-se também a importância dos serviços de saúde, bem como de seus profissionais, no manejo do tratamento de pacientes com paralisia cerebral em suas equipes multi e interdisciplinares, que prestam assistência a essa população, na valorização das ações preventivas, orientações aos cuidadores e medidas precoces de intervenção, visando prevenir possiveis complicações e reconhecer os sinais e sintomas, melhorando a qualidade de vida dessa população.

\section{CONCLUSÃO}

Identificou-se, nesta revisão integrativa, que os principais fatores de risco para a pneumonia aspirativa em crianças com paralisia cerebral foram, respectivamente, com maior prevalência, a aspiração, disfagia, refluxo, sialorreia, Grau V do GMFCS, penetração, sintomas respiratórios durante as refeições, sintomas respiratórios frequentes, ronco noturno e convulsão. 0s temas mais abordados pelos estudos foram aspiração e disfagia.

Apontam-se como lacunas do conhecimento a ausência de estudos que abordem medidas preventivas na pneumonia aspirativa em crianças com paralisia cerebral e estudos sobre orientações domiciliares para cuidadores dessa população. Enfatiza-se a baixa evidência dos artigos encontrados e a necessidade de realização de ensaios clínicos sobre a temática. Percebe-se, além disso, a escassez de pesquisas brasileiras diante da alta prevalência de novos casos no território nacional.

Sugere-se, portanto, a realização de estudos com alta evidência científica que abordem os fatores de risco para as pneumonias aspirativas em crianças portadoras de paralisia cerebral, bem como a aplicação de medidas preventivas que incluam abordagens educativas para cuidadores em âmbito domiciliar.

\section{CONTRIBUIÇÃO DOS AUTORES}

\section{Ligia Rodrigues Carneiro Vasconcelos} contribuiu com a realização da pesquisa, o delineamento do estudo, a redação e a revisão crítica do manuscrito. Roberto Wagner Júnior Freire de Freitas contribuiu com o delineamento do estudo, a redação e a revisão crítica do manuscrito.

\section{REFERÊNCIAS}

1. Brasil. Diretrizes de Atenção à Pessoa com Paralisia Cerebral [serial on the internet]. Brasília (DF): Ministério da Saúde; 2014. Available from: http://bvsms.saude.gov.br $\angle$ bvs/publicacoes/diretrizes atencao paralisia cerebral.pdf 
2. Farias $B H L$, Penha CS, Marques $P C$, Sobreira FA, Teles NHC, Silva GPF, et al. Classificação da função motora grossa em crianças com paralisia cerebral assistidas por um centro por um centro especializado de reabilitação. Braz J Develop. 2020;6(4):18385392. doi: http://dx.doi.org/10.34117/bjdv6n4-128

3. Gomes GC, Jung BC, Nobre CMG, Norberg PKO, Hirsch CD, Dresch FD. Rede de apoio social da família para o cuidado da criança com paralisia cerebral. Rev Enferm UERJ. 2019;27:1-6. doi: https://doi. org/10.12957/reuerj.2019.40274

4. Borges MBS, Galigali AT, Assad RA. Prevalência de distúrbios respiratórios em crianças com paralisia cerebral na Clínica Escola de Fisioterapia da Universidade. Fisioter Mov [serial on the internet]. 2017;18(1):47. Available from: https://periodicos.pucpr.br/ index.php/fisio/article/view/18537/17970

5. Siriwat R. Mechanical Insufflation-Exsufflation Versus Conventional Chest Physiotherapy in Children With Cerebral Palsy. Respir Care. 2018;63(2):18793. doi: https://doi.org/10.4187/respcare.05663

6. Mendes KDS, Silveira RC, Galvão CM. Revisão integrativa: método de pesquisa para a incorporação de evidências na saúde e na enfermagem. Texto Contexto Enferm. 2008;7(4):758-64. doi: https:// doi.org/10.1590/S0104-07072008000400018

7. Lockwood C, Porrit K, Munn Z, Rittenmeyer L, Salmond S, Bjerrum M, et al. Chapter 2: Systematic reviews of qualitative evidence. In: Aromataris $E$, Munn Z, editors. Adelaide: Joanna Briggs Institute; 2017. doi: https://doi.org/10.46658/JBIMES-20-03

8. Rania E. Fundamentos de pesquisa clínica. Porto Alegre: AMGH; 2015.

9. Galvão TF, Pansani TSA. Principais itens para relatar Revisões sistemáticas e Meta-análises: A recomendação PRISMA. Epidemiol Serv Saude. 2015;24(2):335-342. doi: https://doi.org/10.5123/ S1679-49742015000200017

10. Tanaka N, Nohara K, Ueda A, Katayama T, Ushio $M$, Fujii N, et al. Effect of aspiration on the lungs in children: acomparison using chest computed tomography findings. BMC pediatr. $2019 ; 19(162)$. doi: https://doi.org/10.1186/s12887-019-1531-6

11. Baikie G, Reddihough DS, South M, Cook DJ. The salivagram in severe cerebral palsy and able-bodied adults. J Paediatr Child Health. 2009;45(6). doi: https://doi.org/10.1111/j.1440-1754.2009.01496.x

12. Shay E0, Meleca JB, Anne S, Hopkins B. Natural history of silent aspiration on modified barium swallow studies in the pediatric population. International journal of pediatric otorhinolaryngology. Int $\mathrm{J}$
Pediatr Otorhinolaryngol. 2019;125:116-21. doi: https://doi.org/10.1016/j.ijporl.2019.06.035

13. Furkim AM, Duarte ST, Sacco AFB, Sória FS. 0 uso da ausculta cervical na inferência de aspiração traqueal em crianças com paralisia cerebral. Rev CEFAC. 2009;11(4). doi: https://doi.org/10.1590/ S1516-18462009000800011

14. Hafifah CN, Setyanto DB, Putra ST, Mangunatmadja I, Wulandari HF, Sari TT. Gross motor dysfunction as a risk factor for aspiration pneumonia in children with cerebral palsy. PI. 2018;57(5):229-3. doi: https://doi.org/10.14238/ pi57.5.2017.229-33

15. Gerdung CA, Tsang A, Yasseen AS, Armstrong K, Mcmillan HJ, Kovesi T. Association Between Chronic Aspiration and ChronicAirway Infection with Pseudomonas aeruginosa and Other GramNegative Bacteria in Children with Cerebral Palsy. Lung. 2016;194(2). doi: https://doi.org/10.1007/ s00408-016-9856-5

16. Manrique D, Sato J. Salivary gland surgery for control of chronic pulmonary aspiration in children with cerebral palsy. Int J Pediatr 0torhinolaryngol. $2009 ; 73(9)$. doi: https://doi.org/10.1016/j. ijporl.2009.05.002

17. Noonan K, Prunty S, Ha JF, Vijayasekaran S. Surgical management of chronic salivary aspiration. Int J Pediatr Otorhinolaryngol. 2014;78(12). doi: https://doi.org/10.1016/j.ijporl.2014.09.008

18. Weir KA, Mcmahon S, Taylor S, Chang AB. Oropharyngeal aspiration and silent aspiration in children. Chest. 2011;140(3). doi: https://doi. org/10.1378/chest.10-1618

19. Sullivan PB, Morrice JS, Vernon-Roberts A, Grant H, Eltumi M, Thomas AG. Does gastrostomy tube feeding in children withcerebral palsy increase the risk of respiratory morbidity? Arch Dis Child. 2006;91(6). doi: https://doi.org/10.1136/ adc.2005.084442

20. Blackmore AM, Bear N, Blair E, Langdon K, Moshovis L, Steer $\mathrm{K}$, et al. Predicting respiratory hospital admissions in young people with cerebral palsy. Arch Dis Child. 2018;103(12):1119-24. doi: https://doi.org/10.1136/archdischild-2017-314346

21. Arslan SS, Demir N, Karaduman AA. Both pharyngeal and esophageal phases of swallowing are associated with recurrent pneumonia in pediatric patients. Clin Respir J. 2018;12(2). doi: https://doi.org/10.1111/crj.12592

22. Blackmore AM, Bear N, Blair E, Gibson N, Jalla C, Langdon K, et al. Factors associated with respiratory Illness in children and young adults 
with cerebral palsy. J Pediatr. 2016;168. doi: https://doi.org/10.1016/j.jpeds.2015.09.064

23. Zamir 0, Udassin R, Seror D, Vromem A, Freund HR. Laparoscopic Nissen fundoplication in children under 2 years of age. Surg Endosc. 1997;11(12). doi: https://doi.org/10.1007/s004649900569

24. Gustafsson PM, Tibbling L. Gastro-oesophageal reflux and oesophageal dysfunction in children andadolescents with brain damage. Acta paediatrica. 1994;83(10). doi: https://doi. org/10.1111/j.1651-2227.1994.tb12990.x

25. Gubbay A, Blackmore AM. Effects of salivary gland botulinum Toxin-A on drooling and respiratory morbidity in children with neurological dysfunction. Int $\mathrm{J}$ Pediatr Otorhinolaryngol. 2019;124:124-28. doi: https://doi.org/10.1016/j. ijporl.2019.05.044

26. Gok G, Cox N, Bajwa J, Christodoulou D, Moody A, Howlett DC. Ultrasound-guided injection of botulinum toxin $A$ intothe submandibular gland in children and young adults with sialorrhoea. $\mathrm{Br} \mathrm{J}$ Oral Maxillofac Surg. 2013;51(3). doi: https://doi. org/10.1590/0100-3984.2015.0056

27. Fung CW, Khong PL, To R, Goh W, Wong V. Videofluoroscopic study of swallowing in children with neurodevelopmental disorders. Pediatr Society. 2004;46(1). doi: https://doi.org/10.1111/j.1442200X.2004.t01-1-.x

28. Kim BR, Sung IY, Choi KH, Kim LS, Ryu JS. Long-term outcomes in children with swallowing dysfunction. Dev Neurorehabil. 2014;17(5):298305. doi: https://doi.org/10.3109/17518423.2013. $\underline{770102}$

29. Setter CM, Mutou F, Bottoni A. Gerenciamento de complicações respiratórias na paralisia cerebral. Rev cient UMC. 2019;4(2). Available from: http:// seer.umc.br/index.php/revistaumc/article/ view $/ 330$

30. Queiroz DTS, Fernandes ACN, Carvalho MS, Silva GG, Muller AB. Comparação entre GMFCS e CIF na avaliação da funcionalidade na paralisia cerebral. Rer Neurocienc. 2020;28:1-27. doi: https://doi. org/10.34024/rnc.2020.v28.10972

31. Dias BLS, Fernandes AR, Filho HSM. Sialorreia em crianças com paralisia cerebral. J Pediatr. 2016;92(6). doi: https://doi.org/10.1016/j. jped.2016.03.006

32. Castilho LS, Rampi CM, Cruz AJS, Lages FS, Leão DM, Abreu MHNG. Gastroesophageal reflux disease in patients with developmental disabilities. Rev Eletr Extensão. 2020;17(36):22-32. doi: https:// doi.org/10.5007/1807-0221.2020v17n36p22
33. Rogers B. Feeding method and health outcomes of children with cerebral palsy. J Pediatr. 2004;145(2). doi: https://doi.org/10.1016/j. jpeds.2004.05.019

34. Santana S, Santana WC, Costa L, Silva AF, Vanin $\mathrm{PH}$. Prevalência de complicações respiratórias em crianças com paralisia cerebral atendidas pela associação Pestalozzi de Maceió e seus desfechos. Cienc Biol Saúde Unit [serial on the internet]. 2017;4(1):11-22. Available from: https:// periodicos.set.edu.br/fitsbiosaude/article/ view $/ 4522 / 2613$

35. Oliveira DFG, Cavalcante DRA, Feitosa SG. Qualidade de vida dos pacientes com câncer oral: Revisão integrativa da literatura. SANARE (Sobral, 0nline) [serial on the internet]. 2020 Jan-June;19(1):121-30. Available from: $\quad$ https://sanare.emnuvens.com.br/ sanare/article/view/1313/722
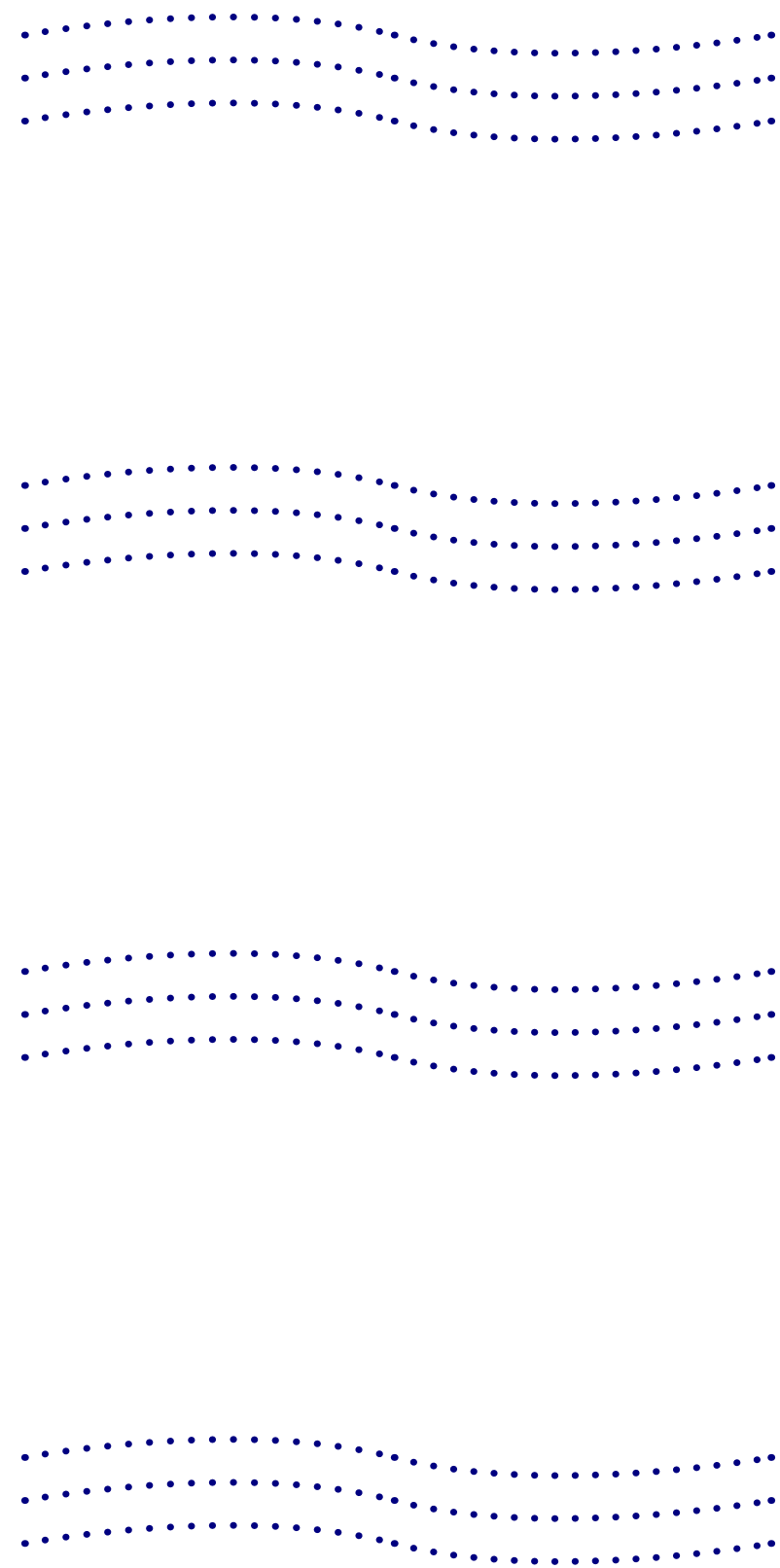